\title{
Effect of pressure, temperature, and magnetic field on the binding energy of the electron-hole system in III-V group semiconductors
}

\author{
Pınar BAŞER ${ }^{1, *(1 D)}$ \\ ${ }^{I}$ Sivas Cumhuriyet University, Department of Physics, Sivas/TURKEY
}

\begin{abstract}
In this study, ground state binding energy of heavy hole magneto exciton in $\mathrm{GaAs} / \mathrm{In}_{0.47} \mathrm{Ga}$ ${ }_{0.53}$ As cylindrical quantum well wires (CQWWs) were calculated using variational technique depending on wire size and external parameters. We can briefly state the change of binding energy with hydrostatic pressure, temperature, wire radius and external magnetic field strength as follows. With increasing temperature for constant pressure and magnetic field, the exciton binding energy decreases slightly. On the other hand, increasing magnetic field strength and pressure increase the binding energy as the particle's quantum confinement effects increase. To interpret these results, we examined pressure and temperature changes of barrier heights, effective masses, wire radius, dielectric constant, and band offsets. Conduction and valence band offset increase by $37 \%$ with pressure, while band offsets decrease by $-1.55 \%$ with temperature. These differences in values are directly due to the difference in pressure and temperature coefficients of the prohibited band gaps of GalnAs and InAs. These variations in binding energy, as well as in electron and hole energies, depending on structure parameters and external parameters provide a prediction to produce adjustable semiconductor devices.
\end{abstract}

\section{Article info}

History:

Received: 01.02.2021

Accepted: 26.04.2021

Keywords:

Quantum Wires;

Exciton Binding

Energy; III-IV

Semiconductor

\section{Introduction}

Studying electron behavior trapped in one-dimensional structures is interesting in terms of solid-state physics and device applications. It is known that quantum well wires (QWWs) structures are used to improve the performance of lasers and nonlinear devices and in new optoelectronic device designs. Advances in crystal growth techniques have allowed the physical realization of semiconductor one dimensional (1D) QWWs structures on nanometer scale. The determination of the Coulombic interaction term, which consists of a confinement of electrons and holes in one-dimensional quantum wires, is very important since it gives information about the optical properties of the structure. Since the particle motion in QWWs structures is free in one dimension, the exciton binding energy and exciton oscillator size increase considerably compared to quantum well (QW) structures where particle since the QWWs particle is confined in two dimensions, it can be said that the exciton binding energy increases significantly compared to QW structures where the particle motion is restricted in one dimension [1]. These semiconductors materials have an important place in device applications. For this reason, both exciton and impurity levels have an important place in determining their optical properties.

The electron hole pair connected to each other by the Coulomb interaction is called an exciton. Numerous experimental and theoretical studies have been carried out showing that the confinement effects of excitonic levels in low-dimensional structures change with applied external fields, hydrostatic pressure, and temperature, and excitonic levels in low-dimensional structures have contributed to optoelectronic device designs [2-6].

Since the symmetry of the system in an external electric and magnetic field decreases, level divisions and new transitions are seen in the spectrum of the exciton. In addition, the ionization and stabilization of exciton states originating from new fields, the formation of diamagnetic bands and the like are observed in the energy spectrum. The effects of external fields on low dimensional structures have been studied extensively [7-12].

Similar to the external magnetic field, hydrostatic pressure and temperature applied to nano structures are external parameters that change the properties of lowdimensional systems. For example, depending on the 
direction of the magnetic field, another electronic confinement occurs in addition to the spatial confinement. Therefore, new electronic properties arise depending on the magnetic field. Since the band gap of the material can be changed by changing these external parameters applied to optoelectronic devices, the semiconductor properties are also changed.

It is an important effect that changes the electronic properties of semiconductors at hydrostatic pressure, such as external fields. Since the pressure changes the lattice parameters and therefore the average distance between electrons and holes, the forbidden band gap and the periodic potential of the structure change with the applied pressure. Similarly, it alters the electronic structure of the semiconductor with temperature. For these reasons, pressure and temperature effects have been studied. The observed temperature-dependent energy range is consistent with the empirical Varshni equation [13]. For these reasons, it has been the focus of attention to examine the properties of $\mathrm{GaAs} / \mathrm{GaAlAs}$ and $\mathrm{GaAs} / \mathrm{InGaAs}$ semiconductors to these external parameters [14].

Structures consisting of $\operatorname{In}_{x} \mathrm{Ga}_{1-\mathrm{x}}$ As ternary compound grown on GaAs substrate are of great interest due to their applications in micro and optoelectronics. $\operatorname{In}_{\mathrm{x}} \mathrm{Ga}_{1}$ ${ }_{x}$ As is a ternary semiconductor compound with direct bandwidths ranging from $0.36 \mathrm{eV}$ to $1.42 \mathrm{eV}$ [15-18]. Some application areas of this semiconductor compound are light emitting diodes (LEDs), laser diodes (LDs) [19-22], quantum well infrared photo detectors (QWIP) [23, 24], high electron mobility transistor (HEMT) devices [25], as well as medium to mid-infrared light sources [26]. Characterization studies for these materials date back to 1978 [27, 28].

Since the lattice constants of GaAs, InP and $\mathrm{d}$ InAs are $5.65,5.86$, and $6.05 \AA$, respectively, the ability to grow thick, high-quality epitaxial layers of $\mathrm{In}_{\mathrm{x}} \mathrm{Ga}_{1-\mathrm{x}}$ As on a GaAs, InP, or InAs substrate is very limited due to Where $|\overrightarrow{\mathrm{r}}|=\left|\vec{r}_{e}-\vec{r}_{h}\right|$ the relative is coordinate, $\vec{P}_{e}$ and $m_{e}^{*}(P, T)$ define the momentum operator and the effective mass of the electron, respectively, while $\vec{P}_{h}$ and $m_{h}^{*}(P, T)$ are the momentum operator and the

$V_{e(h)}\left(\rho_{e(h)}, P, T\right)=\left\{\begin{array}{cc}V_{0 e(h)}(P, T) & \rho_{e(h)} \geq R(P) \\ 0, & \rho_{e(h)}<R(P)\end{array}\right.$

Where $\mathrm{R}(\mathrm{P})$ is the wire radius of CQWWs, $V_{0 e}(P, T)$ is the conduction band offset, $V_{0 h}(P, T)$ is the valans band offset. Band offsets of the GaAs/ $/ \mathrm{In}_{0.47} \mathrm{Ga}_{0.53} \mathrm{As}$ heterostructure for the conduction and valence band, lattice mismatch except for a specific composition. For example, only $\mathrm{In}_{0.53} \mathrm{Ga}_{0.47}$ As lattice matches to $\mathrm{InP}$, and thus very good quality thick films of this composition can be grown on InP [29-31].

One-dimensional nanostructures have great potential not only for basic research materials due to their unique structural and physical properties relative to their ingot structures, but also for future technological applications. The one-dimensional motion of the particle in CQWWs lasers results in improved static and dynamic laser performance. The very low threshold current seen in these lasers will be useful for optoelectronic device and signal applications. With this motivation, the aim of this study is to investigate the effect of magnetic field, hydrostatic pressure, temperature, and wire radius on the binding energy of exciton levels in $\mathrm{GaAs} / \mathrm{In}_{0.47} \mathrm{Ga}_{0.53}$ As one dimensional QWWs. Variational method and effective mass approach were used in these calculations.

\section{Materials and Methods}

The Hamilton equation of the exciton under the magnetic field applied in the $\mathrm{z}$ direction, which is the growth direction of the hetero structure, is defined as in Eq. 1. The solution is made under the variational technique and effective mass approach, considering the changes under pressure and temperature [32]. Theoretical calculations also use cylindrical gauge $\vec{A}_{e(h)}(\vec{r})=(\vec{B} \times \vec{r}) / 2$, and parabolic band approaches. Here the symbol e represents the electron, and the symbol $\mathrm{h}$ indicates the hole.

$$
\begin{aligned}
H= & \frac{1}{2 m_{e}^{*}(P, T)}\left(\vec{P}_{e}+\frac{e}{c} \vec{A}_{e}\right)^{2}+\frac{1}{2 m_{h}^{*}(P, T)}\left(\vec{P}_{h}-\frac{e}{c} \vec{A}_{h}\right)^{2} \\
& +V_{e}\left(\rho_{e}, P, T\right)+V_{h}\left(\rho_{h}, P, T\right)-\frac{e^{2}}{\varepsilon(P, T)|\vec{r}|}
\end{aligned}
$$

effective mass of the hole depending on pressure and temperature, respectively. $\varepsilon(P, T)$ is the dielectric constant and $V_{e(h)}\left(\rho_{e(h)}, P, T\right)$ is the confining potentials, defined as [33]

$70 \%$ (30\%) of the total band gap difference is taken as [34]. Schematic representation of the cylindrical quantum wire in Fig. 1 (a), in Fig.1 (b), the potential profile consisting of the sum of the parabolic confinement caused by the magnetic field and the spatial confinement is shown. 
(a)

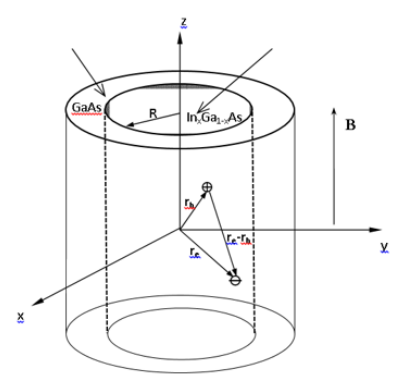

(b)

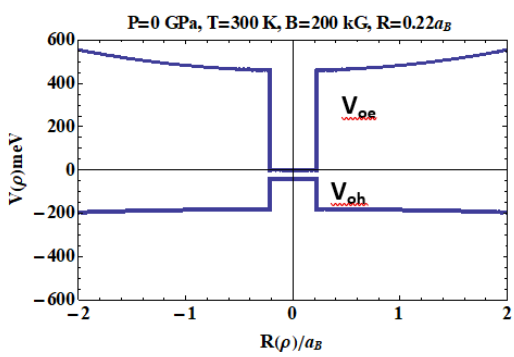

Figure 1. (a) Schematic representation of a cylindrical quantum wire (b) Spatial and parabolic confinement potential for $\mathrm{P}=0 \mathrm{GPa}, \mathrm{T}=300 \mathrm{~K}, \mathrm{~B}=200 \mathrm{kG}$.

Pressure dependence of wire radius it can be derived from fractional change in volüme

$\left(\Delta V / V_{0}=\left(-3 P\left(S_{11}+S_{12}\right)\right)\right.$ due to change in hydrostatic pressure is given by [35],

$R(P)=R(0)\left[1-3 P\left(S_{11}+2 S_{12}\right)\right]^{1 / 2}$

$\mathrm{R}(0)$ is the original wire radius at $\mathrm{P}=0 \mathrm{GPa}$. Here, $S_{11}$ and $S_{12}$ are elastic constants. It can be defined as $|\overrightarrow{\mathrm{r}}|$ in the Hamiltonian equation.

$|\vec{r}|=\left|\vec{r}_{e}-\vec{r}_{h}\right|=$

$\sqrt{\rho_{e}^{2}+\rho_{h}^{2}-2 \rho_{e} \rho_{h} \cos \left(\phi_{e}-\phi_{h}\right)+\left(z_{e}-z_{h}\right)^{2}}$

Since the difference between the effective mass and dielectric constants, which are among the parameters changing due to temperature and pressure, is very small, the values of GaAs semiconductor were used in the calculations of GaAs and GaInAs. Parameter values of $\mathrm{GaAs}$ were used in calculations $\left[m_{e}^{*}=0.0665\right.$ $\mathrm{m}_{0}, m_{h}^{*}=0.134 \mathrm{~m}_{0}, \mathrm{~m}_{0}$ is the free electron mass, and $\varepsilon=12.4$ ] [36]. It is given as the electron mass depending on external parameters.

$m_{e}^{*}(P, T)=\left[1+E_{P}^{\Gamma}\left(\frac{2}{E_{g}^{\Gamma}(P, T)}+\frac{1}{E_{g}^{\Gamma}(P, T)+0.341}\right)\right]^{-1} m_{0}$

Where $E_{P}^{\Gamma}=7.51 \mathrm{eV}$. We used the isotropic heavy hole mass here. For a heavy hole, the effective mass depends solely on hydrostatic pressure and is given by $m_{h}^{*}(P)=\left[0.134+\left(a_{2} P+a_{3} P^{2}\right)\right] m_{o}$ (6)

where $a_{2}=-0.1 \times 10^{-2} \mathrm{GPa}^{-1}$ and $a_{3}=5.5 \times$ $10^{-4} \mathrm{GPa}^{-2}$. The heavy hole isotropic hole mass is calculated from

$$
\left(m_{h}^{*}\right)^{-1}=\left(\frac{2}{3}\right)\left(m_{h}^{*}(x, y)\right)^{-1}+\left(\frac{1}{3}\right)\left(m_{h}^{*}(z)\right)^{-1}
$$

$\varepsilon(P, T)$ is defined by [36]

$$
\begin{aligned}
& \varepsilon(P, T)= \\
& \left\{\begin{array}{c}
12.74 \mathrm{e}^{-1.67 * 10^{-2} P} e^{9.4 * 10^{-5}(T-75.6)}, T \leq 200 K \\
13.18 e^{-1.73 * 10^{-2} P} e^{20.4 * 10^{-5}(T-300)}, T>200 K
\end{array}\right.
\end{aligned}
$$

$E_{g}^{\Gamma}(P, T)$, temperature, pressure dependent band gap at $\Gamma$ points is given by

$E_{g}^{\Gamma}(P, T)=E_{g}^{0}+\alpha P-\beta T^{2}(T+c)^{-1}$

Where $E_{g}^{0} \mathrm{r}$ is the forbidden band gap at room temperature without pressure, $E_{g_{(G a A s)}}^{0}=1.52 \mathrm{eV}$, $E_{g_{(I n A s)}^{0}}^{0}=0.42 \mathrm{eV}, \quad \alpha \quad$ is the pressure coefficient $\alpha_{(\mathrm{GaAs})}=10.8 \times 10^{-2} \mathrm{eV} / \mathrm{GPa}, \alpha_{(\operatorname{InAs})}=$ $\left.7.7 \times 10^{-2} \mathrm{eV} / \mathrm{GPa}\right), \quad \beta$ and $\mathrm{c}$ are temperature coefficients, $\beta_{(G a A s)}=5.405 \times 10^{-4} \mathrm{eV} / \mathrm{K}, \beta_{(\text {InAs })}=$ $\left.4.19 \times 10^{-4} \mathrm{eV} / \mathrm{K}\right)$ and $c_{(\mathrm{GaAs})}=204 \mathrm{~K}, c_{(\operatorname{InAs})}=271 \mathrm{~K}$ [37, 38].

The band gap $E_{g}(P, T)$ equation for the $\operatorname{In}_{\mathrm{x}} \mathrm{Ga}_{1-\mathrm{x}} \mathrm{As}$ compound can be written as follows [39].

$$
\begin{aligned}
E_{g}^{\operatorname{GaInAs}}(P, T)= & E_{g}^{\operatorname{GaAs}}(P, T)+\left[E_{g}^{\operatorname{InAs}}(P, T)-\right. \\
& \left.E_{g}^{\operatorname{GaAs}}(P, T)\right] x-0.475 x(1-x)(10)
\end{aligned}
$$

The Hamiltonian eigenfunctions in the absence of the interaction term are defined by confluent hypergeometric functions. In the wave function equation, while $\mathrm{N}$ is the normalization constant, $\left({ }_{1} F_{1}\left(-a_{01}, 1, \xi\right)\right.$ and $U\left(-a_{01}^{\prime}, 1, \xi\right)$ are hypergeometric functions that describe the particle in the InGaAs material within the GaAs material, respectively.

$\psi_{1}\left(\vec{r}_{e}, \vec{r}_{h}\right)$ 


$$
=\left\{\begin{array}{cc}
\operatorname{Nexp}\left(-\frac{\xi_{e}+\xi_{h}}{2}\right){ }_{1} F_{1}\left(-a_{o 1(e)}, 1, \xi_{R(e)}\right)_{1} F_{1}\left(-a_{o 1(h)}, 1, \xi_{R(h)}\right) & \rho_{e(h)} \leq R(P) \\
N \frac{{ }_{1} F_{1}\left(-a_{o 1(e), 1, \xi_{R(e)}}\right)}{U\left(-a_{o 1(e)}^{\prime}, 1, \xi_{R(e)}\right)} \exp \left(-\frac{\xi_{e}+\xi_{h}}{2}\right) U\left(-a_{o 1(e)}^{\prime}, 1, \xi_{e}\right)_{1} F_{1}\left(-a_{o 1(h)}, 1, \xi_{h}\right) & \rho_{e}>R(P) \text { and } \rho_{h} \leq R(P) \\
N \frac{{ }_{1} F_{1}\left(-a_{\left.o 1(h), 1, \xi_{R(h)}\right)}\right.}{U\left(-a_{o 1(h)}^{\prime}, 1, \xi_{R(h)}\right)} \exp \left(-\frac{\xi_{e}+\xi_{h}}{2}\right)_{1} F_{1}\left(-a_{o 1(e)}^{\prime}, 1, \xi_{e}\right)_{1} F_{1}\left(-a_{o 1(h)}, 1, \xi_{h}\right) & \rho_{e} \leq R(P) \text { and } \rho_{h}>R(P) \\
N \frac{{ }_{1} F_{1}\left(-a_{o 1(e)}, 1, \xi_{R(e)}\right)}{U\left(-a_{o 1(e)}^{\prime}, 1, \xi_{R(e)}\right)} \frac{{ }_{1} F_{1}\left(-a_{o 1(h)}, 1, \xi_{R(h)}\right)}{U\left(-a_{o 1(h)}^{\prime}, 1, \xi_{R(h)}\right)} \exp \left(-\frac{\xi_{e}+\xi_{h}}{2}\right) U\left(-a_{o 1(e)}^{\prime}, 1, \xi_{e}\right){ }_{1} F_{1}\left(-a_{o 1(h)}, 1, \xi_{h}\right), & \rho_{e(h)}>R(P)
\end{array}\right.
$$

Here the cyclotron radius $\alpha_{c[e(h)]}$ is defined by $\alpha_{c[e(h)]}=\left(\sqrt{\rho_{e(h)}^{2} / \mu_{e(h)} \omega_{c[e(h)]}}\right)$ while the variable $\xi_{e(h)}$ is defined by $\xi_{e(h)}=\left(\rho_{e(h)}^{2} / 2 \alpha_{c[e(h)]}^{2}\right)$. Where $\omega_{c[e(h)]}$ is the cyclotron frequency, $\mu$ defines the reduced mass, $\omega_{c[e(h)]}$ is defined by $\omega_{c[e(h)]}=$ $\left(e B / m_{e(h)}^{*}\right)$ and $\mu$ is defined by $\mu=$ $\left(m_{e}^{*} m_{h}^{*} / m_{e}^{*}+m_{h}^{*}\right)$. Here $a_{01}$ and $a_{01}^{\prime}$ are eigenvalues inside and outside the wire radius, respectively, and these eigenvalues can be found by applying the boundary condition $\rho_{\mathrm{e}(\mathrm{h})}=\mathrm{R}$ to wave functions and their derivatives in the Eq. 11. The Hamiltonian's solution in Eq. 1 is given as the product of the term containing the variational function and the radial solutions, considering the exciton term [40]. $\mathrm{N}_{1}$ is the normalization constant

$\psi\left(\vec{r}_{e}, \vec{r}_{h}\right)=\mathrm{N}_{1} \psi_{1}\left(\vec{r}_{e}, \vec{r}_{h}\right) \exp \left(-\lambda\left|\vec{r}_{e}-\vec{r}_{h}\right|\right)$

We can calculate the exciton binding energy in CQWWs from the difference in energy between the state where there is no columbic term and the state of interaction. The energies are scaled to the Rydberg constant, the Bohr radius in lengths (scaled with Rydberg constant for $\mathrm{P}=0, R_{B}=$ $\left(\mu e^{4} / \epsilon_{0}^{2} \hbar^{2}\right)(3.5 \mathrm{meV}$ in GaAs) and Bohr radius, $a_{B}=\left(\epsilon_{0} \hbar^{2} / \mu e^{2}\right)(155 \AA$ in GaAs $\left.)\right)$. Binding energy is given by $E_{b}(R, B, P, T)$ [40],

$$
E_{b}(R, B, P, T)=E_{e 1}+E_{h h 1}-\min _{\lambda}\langle\psi|\widehat{H}| \psi\rangle
$$

Where $E_{e 1}$ and $E_{h h 1}$ are magnetoelectric band energies. $\min _{\lambda}\langle\psi|\widehat{H}| \psi\rangle$ is the minimized value of $\langle\psi|\widehat{H}| \psi\rangle$ with respect to $\lambda$.

\section{Results and Discussion}

The change of the heavy hole exciton binding energy in CQWWs consisting of GaAs-InGaAs heterostructure for different pressure values at room temperature and in the absence of magnetic field, depending on the wire size is given by Fig. 2. In numerical calculations, in concentration is $\mathrm{x}=0.47$, $V_{o e}=124.13, V_{o h}=53.19 \mathrm{meV}$ and $\mu=0.0447 m_{o}[40]$. For all pressure values, we see that the binding energy decreases as the wire radius increases. This is in line with our expectations, because as the wire radius increases the spatial limitation on the particle decreases. In this case, we find the probability density of the particle at any value of the large wire radius. It is clearly seen in Fig. 3 (a). Since the electron and the hole move closer together as the wire radius decreases, with decreasing wire radius the binding energy increases until a certain maximum value is reached. Particles of maximum value are closest to each other. As can be seen from the Fig. 3 (b). With the wire radius decreasing after this maximum value, the binding energy rapidly decreases to the characteristic bulk value of the GaAs. The correctness of this statement is shown in Fig. 3 (c). 


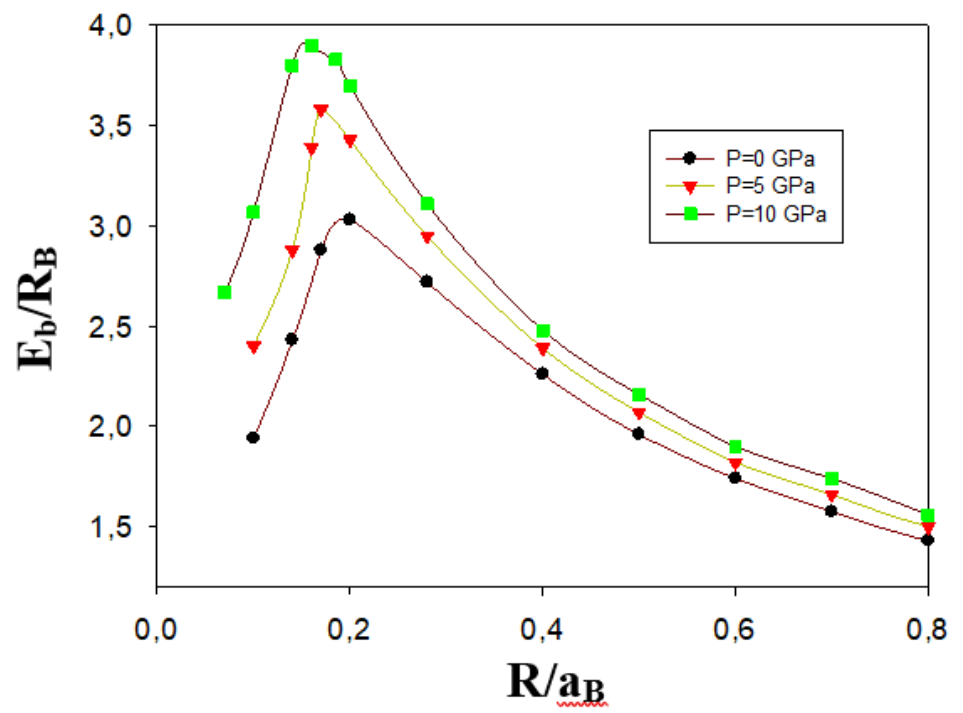

Figure 2. Exciton ground state binding energy for various hydrostatic pressure values

In addition, another point that draws attention is that the pressure value increases, and the maximum binding energy occurs at smaller wire radii. This is in line with our expectation, as the exciton Bohr radius decreases with increasing pressure. The changes in Fig. 2, where

(a)

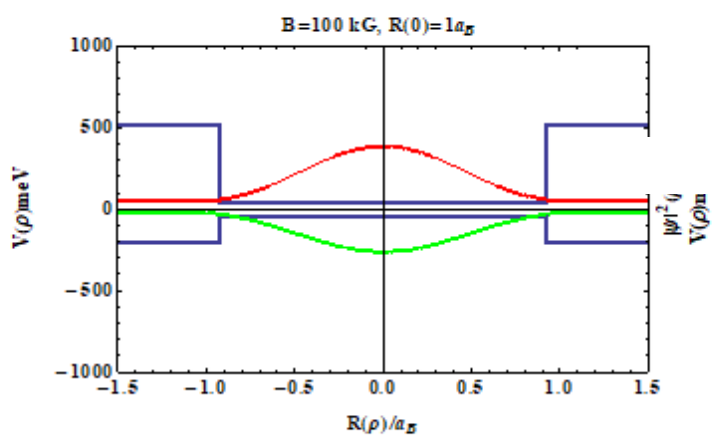

we interpret the increase and decrease of the binding energies depending on the size of CQWWs, can be corrected by the exciton probability distributions plotted at room temperature $\mathrm{P}=10 \mathrm{GPa}$ for a certain wire size in Fig. 3 (a), Fig. 3 (b) and Fig. 3 (c), respectively. (b)

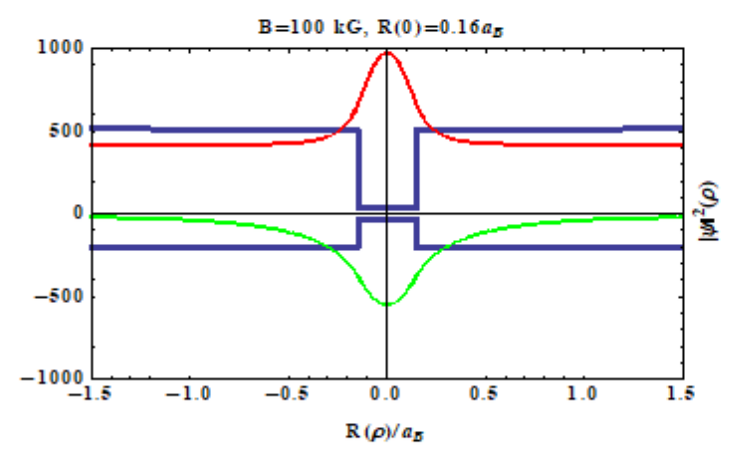

(c)

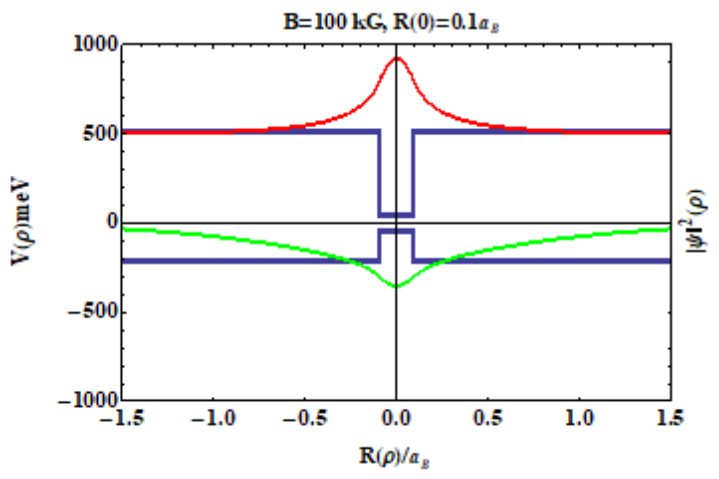

Figure 3. Probability distribution of the heavy hole exciton in the CQWWs on the wire size for (a) $R=1 a_{B}(\mathbf{b}) R=0.16 a_{B}$ (c) $\mathrm{R}=0.1$ aв. 
For the wire radius for $\mathrm{R}=1 \mathrm{a}_{\mathrm{B}}$, the electron and the hole may not be close to each other as the spatial confinement is weakened. For this reason, the binding energy is expected to decrease in large wire radii. (Fig. 3 (a)). In the case of $\mathrm{R}=0.16 \mathrm{a}_{\mathrm{B}}$, the spatial confinement effect is strong, and the particles are closest to each other, so the binding energy is highest (Fig. 3 (b)). Particles from the wire size of $R=0.1 \mathrm{a}_{\text {в }}$ are very energetic and the quantum encirclement effect is very reduced, so the particles appear to leak out of the wire and the binding energy decreases again (Fig. 3 (c)).

In addition, as can be seen from Fig. 2, as the pressure increases at all wire radius values, the binding energy also increases. To illustrate this situation better, we have given the parameter change of the structure according to the pressure for $\mathrm{T}=300 \mathrm{~K}, \mathrm{~B}=0 \mathrm{kG}$ and $\mathrm{R}=0.2 \mathrm{a}_{\mathrm{B}}$ in Table 1. This can be understood from the fact that as the pressure increases, the values of $\mathrm{R}(\mathrm{P})$ and $€(P, T)$ decrease, while the confinement potential, electron effective mass and hole effective mass increase. When the pressure is changed from $0 \mathrm{GPa}$ to $10 \mathrm{GPa}$, the increase in potential heights is $0.37 \%$, while the decrease in the wire radius is $-0.10 \%$.

In this case, as the encircling effects on the particles increase, it is seen that the magnetoelectronic binding energy increases depending on the pressure. Table 2 shows the change of parameters with temperature for $P$ $=0 \mathrm{GPa}, \mathrm{B}=0 \mathrm{kG}$ and $\mathrm{R}=0.2 \mathrm{a}_{\mathrm{B}}$. In this table, potential heights are given in $\mathrm{meV}$.

Table 1. Change of CQWWs parameters with hydrostatic pressure

\begin{tabular}{|c|c|c|c|c|c|c|c|c|c|c|c|c|}
\hline $\mathrm{P}(\mathrm{GPa})$ & $\varepsilon$ & $\begin{array}{l}\varepsilon \\
\%\end{array}$ & $\mathrm{R}(\mathrm{P}) / \mathrm{a}_{\mathrm{B}}$ & $\begin{array}{c}\mathrm{R}(\mathrm{P}) / \mathrm{a} \text { в } \\
\%\end{array}$ & $\mathrm{me}_{\mathrm{e}} / \mathrm{m}_{0}$ & $\begin{array}{c}\mathrm{me}_{\mathrm{e}} / \mathrm{m}_{0} \\
\%\end{array}$ & $\mathrm{~m}_{\mathrm{hh}} / \mathrm{m}_{0}$ & $\begin{array}{c}\mathrm{mh}_{\mathrm{h}} / \mathrm{m}_{0} \\
\%\end{array}$ & $V_{\text {oe }}$ & $\begin{array}{l}\mathrm{V}_{\text {oe }} \\
\%\end{array}$ & $V_{\text {oh }}$ & $\begin{array}{l}\mathrm{V}_{\text {oh }} \\
\%\end{array}$ \\
\hline 0 & 13.18 & $0.00 \%$ & 0.20 & $0.00 \%$ & 0.067 & $0.00 \%$ & 0.134 & $0.00 \%$ & 124.13 & $0.00 \%$ & 53.19 & $0.00 \%$ \\
\hline 5 & 12.08 & $0.22 \%$ & 0.19 & $-0.05 \%$ & 0.087 & $1.49 \%$ & 0.143 & $0.067 \%$ & 146.98 & $0.18 \%$ & 62.99 & $0.18 \%$ \\
\hline 10 & 11.08 & $0.44 \%$ & 0.18 & $-0.10 \%$ & $\begin{array}{c}0 . \\
110\end{array}$ & $\begin{array}{c}- \\
1.49 \%\end{array}$ & 0.180 & $0.34 \%$ & 169.83 & $0.37 \%$ & 72.78 & $0.37 \%$ \\
\hline
\end{tabular}

Table 2. Change of CQWWs parameters with temperature

\begin{tabular}{|c|c|c|c|c|c|c|c|c|}
\hline $\mathrm{T}(\mathrm{K})$ & $\varepsilon$ & $\begin{array}{c}\varepsilon \\
\%\end{array}$ & $\mathrm{~m}_{\mathrm{e}} / \mathrm{m}_{0}$ & $\begin{array}{c}\mathrm{m} / \mathrm{m} 0 \\
\%\end{array}$ & $\mathrm{~V}_{\text {oe }}$ & $\begin{array}{c}\mathrm{V}_{\text {oe }} \\
\%\end{array}$ & $\begin{array}{c}\mathrm{V}_{\text {oh }} \\
\%\end{array}$ & $\begin{array}{c}\mathrm{V}_{\text {oh }} \\
0\end{array}$ \\
\hline 0 & 12.39 & $0.00 \%$ & 0.067 & $0.00 \%$ & 126.08 & $0.00 \%$ & 54.03 & $0.00 \%$ \\
\hline 50 & 12.52 & $0.22 \%$ & 0.066 & $-1.49 \%$ & 126.00 & $-0.06 \%$ & 54.00 & $-0.06 \%$ \\
\hline 100 & 12.65 & $0.44 \%$ & 0.066 & $-1.49 \%$ & 125.77 & $-0.24 \%$ & 53.90 & $-0.24 \%$ \\
\hline 150 & 12.78 & $0.67 \%$ & 0.066 & $-1.49 \%$ & 125.47 & $-0.48 \%$ & 53.77 & $-0.48 \%$ \\
\hline 200 & 12.91 & $1.04 \%$ & 0.065 & $-2.98 \%$ & 125.07 & $-0.80 \%$ & 53.60 & $-0.78 \%$ \\
\hline 300 & 13.18 & $2.07 \%$ & 0.063 & $-5.97 \%$ & 124.13 & $-1.55 \%$ & 53.19 & $-1.55 \%$ \\
\hline
\end{tabular}

With increasing temperature, we would expect the binding energy to decrease because with temperature, the effective mass and confinement potential decrease while the dielectric constant increases. However, as can be seen from Table 2, these change percentages are very low. So, the temperature changes do not affect the binding energy. However, increasing dielectric constant (weaker coulombic interaction) and 
decreasing potential height (less confinement) and effective mass (higher mobility) all contribute to a decrease in binding energy show that the temperature effect is negligible, we plotted the variation of the bond energy with temperature at the wire radius $R=0.3 a_{B}$.

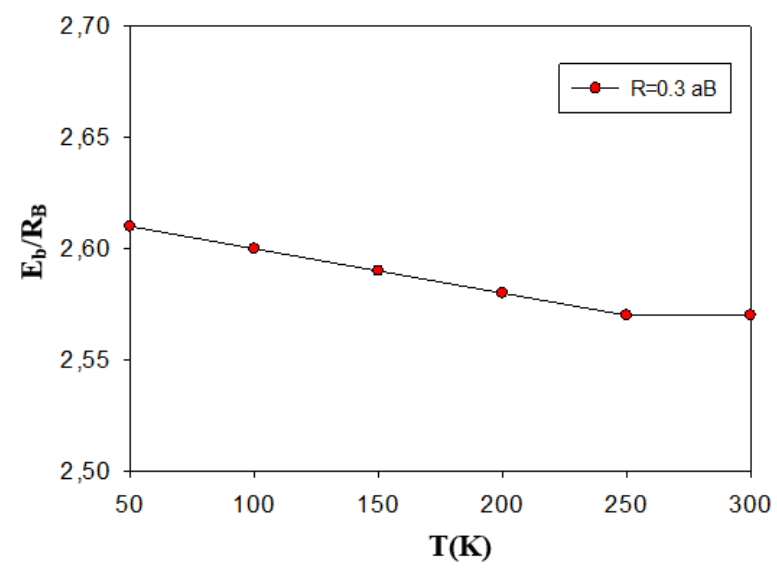

Figure 4. Change of binding energy with temperature at wire radius $\mathrm{R}(0)=0.3 \mathrm{a}_{\mathrm{B}}$

As can be seen from Fig. 4, the percentage change in binding energy in this temperature range is even less than 1.15 percent. To see the percentage changes in Table 1 and Table 2 more clearly, in Fig. 5 (a) and Fig. 5 (b), the effective masses, potential heights for the electron and hole, the wire radius and dielectric constant versus $\mathrm{P}$ and $\mathrm{T}$ versus the total we showed the percentage changes.

(a)

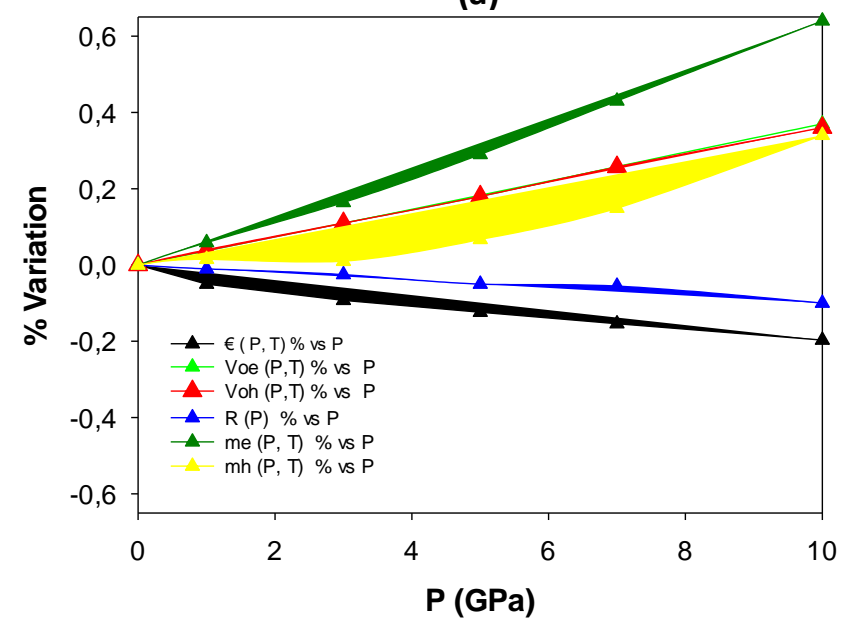

(b)

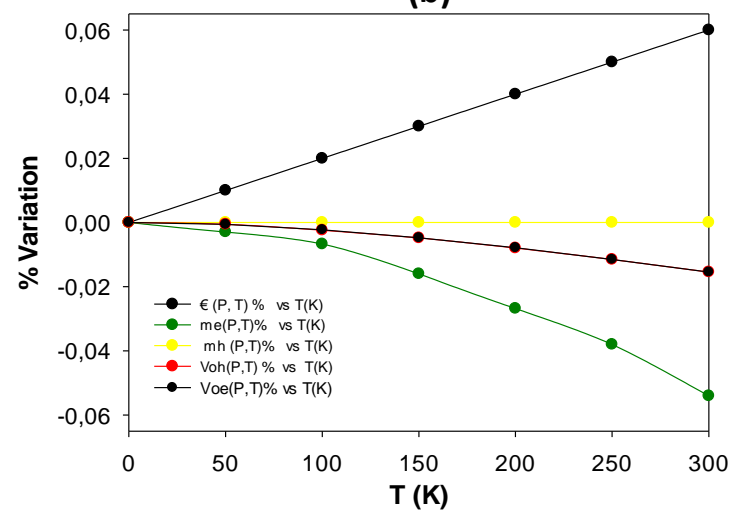

Figure 5. Percentage change of changing parameters with pressure and temperature in GaAs / InGaAs CQWWs (a) vs $\mathrm{P}$ (b) vs $\mathrm{T}$

Lines with triangle symbols show the percentage change with pressure (Fig. 5 (a)), while the lines with circle symbols show the percentage changes of parameters (Fig. 5 (b)) depending on the temperature. It is seen that the change with temperature is much less than the change with pressure. It can be seen from Fig. 5 (b) that the system is very stable under temperature changes. However, Fig. 5 (a) shows that the change of the parameters of the system with pressure is much more than the temperature. Fig. 6 shows how binding energy is affected by the magnetic field at $\mathrm{P}=0 \mathrm{GPa}$ and $\mathrm{T}=300 \mathrm{~K}$.

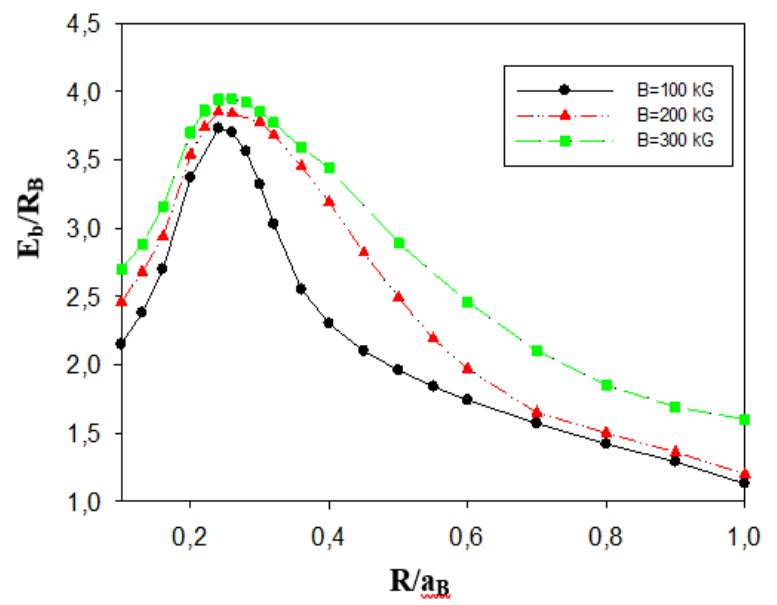

Figure 6. Radius variation of binding energy for different magnetic field sizes

In general, it is seen that the binding energy increases with the increase of magnetic field strength. As the wire radius increases $\left(R \geq 0.5 \mathrm{a}_{\mathrm{B}}\right)$ the spatial envelope decreases and the magnetic envelope is effective on the exciton. In the range of $0.22 \mathrm{a}_{\mathrm{B}}<\mathrm{R}<0.5 \mathrm{a}_{\mathrm{B}}$ for any magnetic field strength, the exciton binding energy begins to increase with decreasing wire radius from the bulk value of InGaAs until it reaches a maximum 
value. In this radius range spatial confinement dominates the binding energy. Finally, the bulk value of $(\mathrm{GaAs})$ drops sharply after reaching a maximum point. This value is approximately $2.23 \mathrm{R}_{\mathrm{B}}$ [38].

\section{Conclusions}

In this study, we calculated the change of exciton binding energy in GaAs / $\operatorname{In}_{0.47} \mathrm{Ga}_{0.53}$ As CQWWs depending on external parameters such as magnetic field, temperature and hydrostatic pressure and wire size. As the wire radius decreases, the bonding energy starts to increase and after reaching a maximum value, it decreases until the ingot GaAs value. The variation of the ground state binding energy depending on external parameters can be summarized as follows. Heavy hole exciton binding energy, depending on hydrostatic pressure and magnetic field. We have seen that due to the increasing hydrostatic pressure and magnetic field, the heavy hole exciton binding energy increases in accordance with the literature. The hydrostatic pressure effect is weaker at large wire radius values. With the increasing temperature under constant pressure and magnetic field, the bonding energy decreases, albeit a little. However, since the temperature-related decrease in the $0-300 \mathrm{~K}$ range is below 1.15 percent, we can say that the bonding energy almost does not change with temperature.

\section{Acknowledgment}

The author would like to thank Sivas Cumhuriyet University for its opportunities.

\section{Conflicts of interest}

The author state that did not have conflict of interests.

\section{References}

[1] Ando H., Nojima, S., Kanbe, H., Band- edge optical absorption spectra of GaAs quantum wires calculated by multiband effective mass theory, Journal of Applied Physics 74 (1993) 6383-6390.

[2] Gurmessa A., Mengesha M., International Journal of Physical Sciences, 14 (7) (2019) 55-61.

[3] Duque C. A, Oliveira L. E, de Dios-Leyva M, correlated electron-hole transitions in bulk GaAs and $\mathrm{GaAs}-(\mathrm{Ga}, \mathrm{Al})$ As quantum wells: effects of applied electric and inplane magnetic fields, Birazilian Journal of Physics, 36 (2006) 10381041.
[4] Pokutnyi S. I., Tyc M. H., Salejda W., Misiewicz J., Two-dimensional WannierMott exciton in a uniform electric field, Physics of the Solid State., 43 (2001) 923- 926.

[5] Raigoza N., Duque C.A., Reyes-Go'mez E., Oliveira L.E., Effects of hydrostatic pressure and applied electric fields on the exciton states in GaAs-(Ga, Al) As quantum wells, Physica B, 367 (2005) 267-274.

[6] Venkateswaran U., Chandrasekhar M., Chandrasekhar H. R., Bojak B. A., Chambers F.A., Meese J. M., Phys. Rev. B, 33 (1986) 84168423.

[7] Saravanan S., John Peter A., Binding Energy of a Magneto-exciton in an InAsP Quantum Well Wire for the Potential Application of Telecommunication Networks, Materials Today: Proceedings, 2 (2015) 4373- 4377.

[8] Vurgaftman I. and Meyer J. R., Ram-Mohan L. R., Band parameters for III-V compound semiconductors and their alloys, Journal of Applied Physics, 89 (2001) 5815 -5875.

[9] Morales A. L., Raigoza N., Duque C. A. and Oliveira L. E., Effects of growthdirection electric and magnetic fields on excitons in GaAs-Ga1-xAlxAs coupled double quantum wells, Phys. Rev. B, 77 (2008) 113309-1-1133094.

[10] Butov L. V., Shashkin A. A., Dolgopolov V. T., Campman K. L., and Gossard A. C., Magnetooptics of the spatially separated electron and hole layers in GaAs/AlxGa1-xAs coupled quantum wells, Phys. Rev. B, 60 (1999) 8753-8758.

[11] Kavokin A. V. Kokhanovski S. I. Nesvizhki A. I. Sasin M. E. Sesyan R. P. Ustinov V. M. Yu. Egorov A. Zhukov A. E. and Gupalov S. V. Semiconductors, 31 (1997) 950-960.

[12] Agekyan V. T., Spectroscopic properties of semiconductor crystals with direct forbidden energy gap, Phys. Stat. Sol. (a), 43 (1977) 11-42.

[13] Varshni Y. P., Temperature dependence of the energy gap in semiconductors, Physica, 34 (1967) 149-154.

[14] Peter A. J., Gnanasekar K., Navaneethakrishnan K., Binding energy of impurity states in a parabolic quantum dot in a strong magnetic field, Phys. Stat. Sol. (b), 242 (2005) 2480-2488.

[15] Di Dio M., Lomascolo M., Passaseo A., Gerardi C., Giannini C., Quirini A., Tapfer L., Giugno P.V., De Vittorio M., Greco D., Convertino A. L., 
Vasanelli L., Rinaldi R., Cingolani R., J. Appl. Phys., 80 (1996) 482-489.

[16] Martini S., Quivy A. A., da Silva E. C. F., Leite J. R., Real-time determination of the segregation strength of indium atoms in InGaAs layers grown by molecular-beam epitaxy, Appl. Phys. Lett., 81 (2002) 2863-2865.

[17] Martini S., Quivy A. A., Tabata A., Leite J. R., Influence of the temperature and excitation power on the optical properties of $\mathrm{InGaAs} / \mathrm{GaAs}$ quantum wells grown on vicinal GaAs (001) surfaces (2001), Journal of Applied Physics, 90 (2001) 2280- 2289.

[18] Bratkovski A. and Kamins T. I., Nanowire-Based Light-Emitting Diodes and Light-Detection Devices with Nanocrystalline Outer Surface, Google Patents, (2010)

[19] Baba T., Yogo Y., Suzuki K., Koyama F., Iga K., Near room temperature continuous wave lasing characteristics of GaInAsP/InP surface emitting laser, Electron. Lett., 29 (1993) 913-914.

[20] Uchida T., Koyama F., and Iga K., Control of GaInAs/InP layer thickness for surface- emitting lasers by chemical beam epitaxy, Electron. Communi. in Japan Part II ,75 (1992) 101-107.

[21] Pyun S. H., Jeong W. G., Korean J., Phys. Soc J. Korean Phys. Soc., 56 (2010) 586- 590.

[22] Soulby M. R., Revin D. G., Commin J. P., Krysa A. B., Roberts J. S., Cockburn J. W., Probing diagonal laser transitions in InGaAs/AlInAs/InP quantum cascade lasers, J. Appl. Phys., 106 (2009) 123106-123109.

[23] Matthews M. R., Steed R. J., Frogley M. D., Phillips C. C., Attaluri R. S. and Krishna S., Transient photoconductivity measurements of carrier lifetimes in an InAs $/ \mathrm{In}_{0.15} \mathrm{Ga}_{0.85}$ As dots-ina-well detector, Appl. Phys. Lett., 90 (2007) 103519-1- 103519-4.

[24] Tsao S., Lim H., Zhang W., and Razeghi M., High operating temperature $320 \times 256320 \times 256$ middlewavelength infrared focal plane array imaging based on an InAs/nGaAs/nAlAsInPInAs/nGaAs/nAlAs/nP quantum dot infrared photodetector, Appl. Phys. Lett., 90 (2007) 201109-1-.201109-3.

[25] Zekentes K., Halkias G., Dimoulas A., Tabata A., Benyattou T., Guillot G., Morante J. R., Peiró F., Cornet A., Georgakilas A., Christou A., Materials problems for the development of InGaAs/InAlAs
HEMT technology, Mater. Sci. Eng. B, 20 (1993) 21-25.

[26] Connolly J. P. and Rohr C., Quantum well cells for thermophotovoltaics, Semicond. Sci. Technol, 18 (2003) 216-220.

[27] Nahory R. E., Pollack M. A., Johnston W. D., and Barns R. L., Band gap versus composition and demonstration of Vegard's law for $\mathrm{In}_{1-\mathrm{x}} \mathrm{Ga}_{\mathrm{x}} \mathrm{AsP}_{1-\mathrm{y}}$ lattice matched to InP, Appl. Phys. Lett., 33 (1978) 659-661.

[28] Fritz I. J., Klem J. F., Schirber J. E., Olsen J. A., and Bonner W. A., InGaAs/GaAs multiple strained- layer structure grown on a latticematched InGaAs substrate wafer, Appl. Phys. Lett. 66 (1995) 1957-1959.

[29] Adachi S., Oe K., Internal strain and photoelastic effects in $\mathrm{Ga}_{1-\mathrm{x}} \mathrm{Al}_{\mathrm{x}} \mathrm{As} / \mathrm{GaAs}$ and $\mathrm{In}_{1-\mathrm{x}} \mathrm{Ga}_{\mathrm{x}} \mathrm{As}_{\mathrm{y}} \mathrm{P}_{1-\mathrm{y}} / \mathrm{InP}$ crystals, J. Appl. Phys. 54, (1983)6620-6627.

[30] Guldner Y., Vieren J. P., Voos M., Delahaye F., Dominguez D., Hirtz J. P., Razeghi M., Quantum Hall effect in $\mathrm{In}_{0.53} \mathrm{Ga}_{0.47} \mathrm{As}-\mathrm{InP}$ heterojunctions with two populated electric subbands, Phys. Rev. B, 33 (1986) 3990-3993.

[31] Lamberti C., Bordiga S., Structural and optical investigation of $\operatorname{InAs}_{\mathrm{x}} \mathrm{P}_{1-\mathrm{x}} / \mathrm{InP}$ strained superlattices, J. Appl. Phys., 83 (1998) 1058-1077.

[32] Elagoz S., Karki H. D., Baser P., Sokmen I., The magnetoexciton binding energy dependency on aluminium concentration in cylindrical quantum wires, Superlatt. and Microstruct., 45 (2009) 506513.

[33] Karki H. D., Elagoz S., Baser P., Amca R., Sokmen I., Barrier height effect on binding energies of shallow hydrogenic impurities in coaxial $\mathrm{GaAs} / \mathrm{Al}_{\mathrm{x}} \mathrm{Ga}_{1-\mathrm{x}} \mathrm{As}$ quantum well wires under a uniform magnetic field, Superlatt. Microstruct., (4) (2007) 227-236.

[34] Ji G., Huang D., Reddy U. K., Henderson T. S., Houdre R., and Morkoç H., Optical investigation of highly strained InGaAs/GaAs multiple quantum wells, Journal of Applied Physics, 62 (8) (1987) 3366-3373.

[35] Peter, Y. Yu., Cardona M., Fundamentals of Semiconductors, Berlin: Springer, (1996) 276290.

[36] Baser P., Karki H. D., Demir I., Elagoz S., The hydrostatic pressure, and temperature effects on the binding energy of magnetoexcitons in 
cylindrical quantum well wires, Superlatt. Microstruct., 63 (2013) 100-109.

[37] Beltran Rios C. L., Porras-Montenegro N., Pressure, and magnetic field effects on the binding energy of excitonic states in single and coupled GaAs-AlGaAs quantum wells, Microelectronics Journal, 36 (2005) 369-373.

[38] Baser P., Pressure, and temperature effects on magnetoelectric band energies in GaAs / $\mathrm{In}_{\mathrm{x}} \mathrm{Ga}_{1}$ ${ }_{x}$ As cylindrical quantum wires, Cumhuriyet Science Journal, 41(3) (2020) 699-705.

[39] Başer P, Altuntas I, Elagoz S, The hydrostatic pressure, and temperature effects on hydrogenic impurity binding energies in $\mathrm{GaAs} / \mathrm{In}_{\mathrm{x}} \mathrm{Ga}_{1}$ ${ }_{x}$ As/GaAs square quantum well, Superlattice Microst., 92 (2016) 210-216.

[40] Baser P., Electronic properties of low dimensional systems: the hydrogenic impurities and excitonic binding energies in cylindrical $\mathrm{Ga}_{1-\mathrm{x}} \mathrm{Al}_{\mathrm{x}} \mathrm{As} / \mathrm{GaAs}$ quantum wires under an externally applied magnetic field, Marmara University, Institute of science PhD. Thesis, (2007). 